\title{
EDITORIAL
}

\section{The Need for Coaches in the Clinical World}

\author{
Robert L. Pbillips, Jr, MD, MSPH
}

American Board of Family Medicine, Lexington, Kentucky

Ann Fam Med 2021;19:194-195. https://doi.org/10.1370/afm.2700.

$\mathrm{F}$ ive papers in this issue could be read with hope and despair about change in clinical care, but I believe they all call for coaches-the need for facilitation in practice transformation. ${ }^{1,2}$ These studies offer important insights about facilitating behavior change, the importance of culture, respecting complexity, and the real risk that our nation's quality payment program is mass delusion. Starting with the most promising, EvidenceNOW is an important, largescale test of change facilitation in frontline practice (Cohen et al). ${ }^{3}$ The integration of an evaluation plan (ESCALATES) and intense intention to learn was certainly part of its secret sauce, producing dozens of useful studies along the way. The manuscript in this issue reports on the role of practice conditions and facilitation on improving blood pressure and smoking cessation, finding that smaller and physician-owned practices are more likely to have sufficient agency and capacity for translating motivations into change and improvement than larger or system-owned practices. The authors find practice facilitation to be an important ingredient for change, but particularly in the latter clinics. This is an important lesson given the rapid shifts in practice ownership and health system consolidation. The paper adds to evidence for federal and state investments in practice facilitation and is also testament to a decade of careful, thoughtful investment by the Agency for Healthcare Research and Quality in learning how to support practice transformation.

Caring for psychologically complex patients with pain and addiction is tough, but Sokol and colleagues found that an interdisciplinary consultation service can help primary care physicians in specific ways and reduce their burden. ${ }^{4}$ Like practice facilitation, pain

Conflicts of interest: author reports none.

\section{CORRESPONDING AUTHOR}

Robert L. Phillips, Jr

American Board of Family Medicine

1648 McGrathiana Parkway, Suite 550

Lexington, KY 40511

bphillips@theabfm.org and addiction supportive services (PASS) are not generally supported by current payment models, but it turns out that they are important for validating frontline clinician decision making and emotions in caring for this difficult patient population. They also give such patients a sense of more control, can lift some of the burden of management decisions, enable boundary setting, and contribute to learning how to reframe visits around patients' functioning, values, and goals rather than their pain or medications. Access to services like PASS, offering coaching, consulting, and visit scripting may be important to increasing availability and quality of care for this important patient population. And we may need different consulting models to support the wide range of primary care practices.

Reducing unnecessary antibiotic prescribing was an explicit system value when I was a medical student. As an effort, it continues to struggle despite an accumulation of research. Perera et al at University of Auckland nicely summarize much of this evidentiary landscape in framing a 3-arm RCT of testing in-office patient messaging. ${ }^{5}$ Specifically, they test 2 ways of potentially reducing patients' expectations for antibiotic treatment of upper respiratory infections using a necessity-concern framework: The first message is about the futility of antibiotics (necessity); the second on the potential for adverse effects (concern). Practice doctors were aware of the study but not involved outside of usual care. Patients in both intervention arms were significantly less likely to expect antibiotics than the control group-but there were no differences in likelihood of receiving a prescription! Less than one-third of patients received such prescriptions (lower than typical), and some factors were associated with higher odds of receiving a prescription and filling it: (1) patients who strongly wanted antibiotics despite intervention ${ }_{i}(2)$ perception that child's illness was severe; or (3) those with ear pain. It will take patient- and physician-focused interventions to further reduce antibiotic prescribing/receiving for upper respiratory infections, but at least now we have 2 more options for reducing patient expectations.

Despite California policies excluding hospitals with high cesarean delivery rates from health insurance 
exchange plans, and a multi-intervention, statewide maternal quality care collaborative, 30\% of hospitals remained above threshold and a handful even increased cesarean birth rates. A mixed method study led by White VanGompel sought to understand reasons for success or failure in lowering cesarean delivery rates by $5 \%$ from baseline or below $24 \%$ of all births. ${ }^{6}$ The most unsettling finding was that surveyed clinicians felt most patients lack sufficient knowledge to make informed decisions about cesarean vs vaginal delivery (38\% in successful hospitals, $29 \%$ in unsuccessful). The study found greater success in hospitals with more family physicians and midwives and where physicians supported midwifery, and that nurses were generally more supportive of best practices than were physicians. Hospitals with more transparency about metrics and willingness to use peer-review of cesarean births were also more successful. Physicians at successful hospitals were also one-third as likely to prefer elective cesarean delivery over vaginal birth for their own families $(4.6 \%$ vs $14.6 \%)$. Key informant interviews pointed to fear (malpractice, bad outcomes) and lack of inter-professional collaboration and communication as barriers to change. Perhaps the loudest message of this study is that more effort is needed to help patients make informed delivery choices. Culture remains a large barrier and is unlikely to change without facilitation.

The TRial of Aggregate Data Exchange for Maintenance of certification and Raising Quality (TRADEMaRQ) attempted to transfer quality measures from health systems or clinic networks with mature quality measurement processes to the American Board of Family Medicine (for which I was the principal investigator). ${ }^{7}$ The goals were to make quality reporting a byproduct of care, reducing quality assessment burden and aligning incentives for quality improvement, namely federal reporting and certification programs. The trial was largely a failure for both goals. Only one health system successfully and reliably transferred quality measures and could demonstrate a relationship between measuring and improving quality. This network already worked aggressively at quality and publicly reported clinician measures. The general failure does not impugn the other systems, rather it suggests that (1) even mature quality efforts may need more timely, reliable, and believable integration with care and be married with support for improvement; and, (2) federal/commercial quality payment programs may have little hope of improving care unless they use measures meaningful in daily care and support local quality improvement facilitation.

To read or post commentaries in response to this article, go to https://www.AnnFamMed.org/content/19/3/194/tab-e-letters.

Key words: practice facilitation; practice change; quality improvement; coaching

Submitted March 31, 2021; accepted March 31, 2021.

\section{References}

1. Phillips RL, Kaufman A, Mold JW, et al. The primary care extension program: a catalyst for change. Ann Fam Med. 2013;11(2):173-178. doi:10.1370/afm.1495

2. Ono SS, Crabtree BF, Hemler JR, et al. Taking innovation to scale in primary care practices: the functions of health care extension. Health Aff (Millwood). 2018;37(2):222-230. doi:10.1377/ hlthaff.2017.1100

3. Cohen DJ, Sweeney SM, Miller WL, et al. Improving smoking and blood pressure outcomes: the interplay between operational changes and local context. Ann Fam Med. 2021;19(3):240-248.

4. Sokol RG, Pines R, Chew A. Multidisciplinary approach for managing complex pain and addiction in primary care: a qualitative study. Ann Fam Med. 2021;19(3):224-231.

5. Perera Al, Thomas MG, Petrie KJ, et al. Reducing expectations for antibiotics in patients with upper respiratory tract infections: a primary care randomized controlled trial. Ann Fam Med. 2021; 19(3):232-239.

6. White VanGompel EC, Perez SL, Datta A, et al. Culture that facilitates change: a mixed-methods study of hospitals engaged in reducing cesarean deliveries. Ann Fam Med. 2021;19(3):249-257.

7. Phillips RL Jr, Peterson L, Palen TE, et al. Clinical quality measure exchange is not easy. Ann Fam Med. 2021;19(3):209-211. 